\title{
Relationship between Random Blood Glucose, Fasting Blood Glucose, and Gensini Score in Patients with Acute Myocardial Infarction
}

\author{
Yuhan Qin, ${ }^{1}$ Gaoliang Yan $\mathbb{D}^{,},{ }^{2}$ Yong Qiao, ${ }^{2}$ Changle Ma, ${ }^{1}$ Juchuan Liu, ${ }^{1}$ \\ and Chengchun Tang $\mathbb{D}^{2}$ \\ ${ }^{1}$ Medical School of Southeast University, Nanjing 210009, China \\ ${ }^{2}$ Department of Cardiology, Zhongda Hospital Affiliated to Southeast University, Nanjing 210009, China \\ Correspondence should be addressed to Chengchun Tang; tangchengchun@hotmail.com
}

Received 24 May 2019; Revised 19 August 2019; Accepted 24 September 2019; Published 15 October 2019

Academic Editor: Lei Ye

Copyright () 2019 Yuhan Qin et al. This is an open access article distributed under the Creative Commons Attribution License, which permits unrestricted use, distribution, and reproduction in any medium, provided the original work is properly cited.

\begin{abstract}
Objective. To examine the relationship between admission random blood glucose (RBG), fasting blood glucose (FBG), and Gensini score in patients with acute myocardial infarction (AMI) to clarify the effects of RBG and FBG on the severity of coronary artery disease. Method. A total of 958 consecutive AMI patients who underwent emergency coronary angiography at the Cardiology Department of Zhongda Hospital (affiliated with Southeast University) were enrolled in this study from January 1, 2016, to December 31, 2018. The Gensini score of each patient was calculated according to the results of coronary angiography. The RBG, FBG, baseline data, hematological indexes, echocardiography parameters, coronary angiography data, and the use of intra-aortic balloon pump (IABP) were recorded. Patients with an RBG level $>11.1 \mathrm{mmol} / \mathrm{L}$ were classified into the stress hyperglycemia group, and those with an FBG level $>7.0 \mathrm{mmol} / \mathrm{L}$ were classified into the elevated FBG group. The Gensini scores in the stress hyperglycemia and elevated FBG groups were compared to those in the control group, and correlations between the RBG and FBG levels and the Gensini scores of AMI patients were evaluated. Independent risk factors for the Gensini score were analyzed by multiple linear and multiple logistic regression analyses. Results. The Gensini scores of the stress hyperglycemia group and the elevated FBG group were higher than those of the control group. RBG and FBG were positively correlated with the Gensini score, and there were significant differences between RBG and FBG in different Gensini score groups. After adjusting for confounding factors, multiple linear regression analysis showed that sex, diabetes, estimated glomerular filtration rate (eGFR), and FBG were independent risk factors for the Gensini score. Multiple logistic regression analysis showed that age and FBG were independent risk factors in group 2 compared to group 1, eGFR and FBG were independent risk factors in group 3, and eGFR and FBG were independent risk factors in group 4. Diabetes and RBG were not independent risk factors for the Gensini score. Conclusion. The Gensini scores of patients in the stress hyperglycemia group and the elevated FBG group were significantly higher than those in the control group. RBG and FBG were positively correlated with the Gensini score in AMI patients, and FBG was an independent risk factor for the Gensini score in AMI patients.
\end{abstract}

\section{Introduction}

Diabetes is an independent risk factor for coronary artery disease (CAD), and CAD is the main cause of death in diabetes patients. Compared to the nondiabetic population, diabetes is associated with a 2-3-fold increase in the risk of cardiovascular disease and mortality due to cardiovascular disease [1]. Diabetics without a history of myocardial infarction and nondiabetic patients with a history of myocardial infarction have approximately the same risks of acute myocardial infarction (AMI) and death from CAD [2]. Patients with diabetes tend to have more extensive, diffuse, calcified, and severe coronary artery lesions [3] and a higher Gensini score than nondiabetics [4]. AMI patients also have a high prevalence of diabetes, and the INTERHEART study, performed in 52 countries, indicated that diabetes 
contributed $10 \%$ of the attributable risk in newly diagnosed AMI patients [5]. The DECODE study indicated that abnormal glucose tolerance also increased the risk of cardiovascular disease and led to a poor prognosis [6]. Patients with an impaired fasting glucose (IFG) level and impaired glucose tolerance (IGT) have more severe coronary artery lesions [7]. Hyperglycemia is also an independent risk factor for both in-hospital and long-term poor prognosis in AMI patients [8]. Hyperglycemia is related to diabetic microvascular complications, including the incidence of diabetic retinopathy and diabetic nephropathy, the latter of which can be reduced by treating hyperglycemia [9]. This study examined the relationships between admission random blood glucose (RBG), fasting blood glucose (FBG), and Gensini score and evaluated the effects of RBG and FBG on the severity of CAD in AMI patients.

\section{Materials and Methods}

2.1. Study Population. A total of 958 consecutive AMI patients who underwent emergency coronary angiography at the Cardiology Department of Zhongda Hospital (affiliated with Southeast University), from January 1, 2016, to December 31, 2018, were enrolled in this study. The study population had an average age of $62.51 \pm 13.49$, and 744 (77.7\%) were male. A total of 265 patients $(27.7 \%)$ suffered stress hyperglycemia, and 589 (61.5\%) had an elevated FBG level. Patients with AMI who underwent emergency coronary angiography and were aged 18-80 years were enrolled. The exclusion criteria were as follows: an allergy to iodine or iodine contrast agent, severe hemodynamic instability, severe liver and kidney dysfunction, severe infectious diseases, malignant tumors, severe blood system diseases, and incomplete data. All patients provided written informed consent before enrollment in the study.

2.2. Research Method. Baseline data, including sex, age, height, weight, systolic blood pressure (SBP), diastolic blood pressure (DBP), and Killip class, as well as previous and personal histories, including hypertension, diabetes, previous myocardial infarction (MI), cerebral infarction, chronic kidney dysfunction (CKD), and smoking history, were recorded. Hematological examination indexes, including RBG, FBG, troponin I (TNI), creatine kinasemuscle/brain (CK-MB), myoglobin (Myo), B-type natriuretic peptide (BNP), routine blood tests, liver and kidney function, serum lipids, echocardiography parameters, medications, coronary angiography data, and the use of an intra-aortic balloon pump (IABP) or coronary artery bypass grafting (CABG), were recorded.

2.2.1. Coronary Angiography. Left and right coronary angiographic examinations were performed via radial artery puncture; multiposition projection was performed according to Judkins' method. The records were diagnosed independently by at least two interventional physicians blinded to the other data of the patients; a third physician performed the analysis when necessary.
2.2.2. Stress Hyperglycemia. The American Diabetes Association (ADA) defines stress hyperglycemia as an FBG level $>6.9 \mathrm{mmol} / \mathrm{L}$ or an $\mathrm{RBG}$ level $>11.1 \mathrm{mmol} / \mathrm{L}$ with no history of diabetes [10]. In this study, we defined an RBG $>11.1 \mathrm{mmol} / \mathrm{L}$ as stress hyperglycemia, regardless of the history of diabetes [10]. RBG was tested once patients entered hospital, and FBG was tested in the morning of the next day.

2.2.3. Gensini Score. The degree of stenosis and the coronary artery lesion site were scored as follows. The degree of stenosis score was multiplied by the lesion site score, and the sum of the lesion score was taken as the final Gensini score [11] (Table 1). The Gensini score was defined by two separate doctors.

2.3. Statistical Analysis. SPSS 19.0 and MedCalc v18.11.3 software were used for all statistical analyses. Count data are expressed as cases and percentages, and the $\chi^{2}$ test was used for analysis. Numerical data are expressed as mean \pm SD and were compared using the independent sample $t$ test. Nonnormally distributed numerical data are expressed as the median and 25th-75th interquartile range and were compared using a rank-sum test. Spearman correlation analysis was used to evaluate the correlations between the RBG, FBG, and Gensini score. Multiple linear regression analysis and logistic regression analysis were performed to examine independent risk factors for Gensini score as continuous variables and hierarchical variables, respectively. In all analyses, $P<0.05$ was taken to indicate statistical significance.

\section{Results}

3.1. Comparison of the Baseline Data, Hematological Indexes, Coronary Angiography Data, and Gensini Score in the Hyperglycemia Group and the Nonhyperglycemia Group. There were significant differences in sex, age, hypertension, diabetes, previous $\mathrm{MI}$, cerebral infarction, CKD, smoking, Killip class I, Killip class IV, BNP, white blood cell (WBC) count, alanine aminotransferase (ALT), RBG, estimated glomerular filtration rate (eGFR), FBG, triglycerides (TG), total cholesterol (TC), high-density lipoprotein-cholesterol (HDL-C), low-density lipoprotein-cholesterol (LDL-C), left ventricular ejection fraction (LVEF), clopidogrel, ticagrelor, angiotensin-converting enzyme inhibitors (ACEI)/angiotensin II receptor blockers (ARBs), IABP, single-vessel lesion, double-vessel lesions, left main lesion, and Gensini score between the hyperglycemia group and the nonhyperglycemia group $(P<0.05)$. There were no significant differences in height, weight, body mass index (BMI), SBP, DBP, Killip class II, Killip class III, TNI, Myo, CK-MB, hemoglobin $(\mathrm{Hb})$, platelets (PLT), aspartate aminotransferase (AST), serum creatinine (Scr), uric acid (UA), aspirin, $\beta$-blocker, statin, calcium channel blocker (CCB), furosemide, antisterone, $\mathrm{CABG}$, triple-vessel lesions, and number of stents between the hyperglycemia group and the nonhyperglycemia group $(P>0.05)$ (Table 2$)$. 
3.2. Comparison of Baseline Data, Hematological Parameters, Coronary Angiography Data, and Gensini Score in the FBG Elevated Group and the Non-FBG Elevated Group. There were significant differences in sex, BMI, diabetes, cerebral infarction, smoking, Killip class I, Killip class IV, TNI, Myo, BNP, WBC, RBG, ALT, AST, FBG, HDL-C, LVEF, clopidogrel, ticagrelor, ACEI/ARB, furosemide, antisterone, IABP, single-vessel disease, triple-vessel disease, number of stents, left main lesion, and Gensini score between the elevated FBG group and the non-FBG elevated group $(P<0.05)$. There were no significant differences in age, height, weight, SBP, DBP, hypertension, previous MI, CKD, Killip class II, Killip class III, CK-MB, Hb, PLT, Scr, eGFR, UA, TG, TC, LDL-C, aspirin, $\beta$-blocker, statin, CCB, CABG, or double-vessel disease $(P>0.05)$ (Table 3$)$.

3.3. Correlation Analysis of RBG and FBG with Gensini Score in AMI Patients. Spearman correlation analysis showed a positive correlation between RBG and Gensini score $(r=0.182, P<0.001)$. In addition, there was a positive correlation between FBG and Gensini score $(r=0.171$, $P<0.001$ ).

According to the quartile method, we divided the patients' scores into four groups based on the Gensini score: group 1, Gensini score $\leq 37$; group 2, $37<$ Gensini score $\leq 60$; group 3, $60<$ Gensini score $\leq 88$; and group 4, Gensini score $>88$. The correlation coefficient between the RBG and the Gensini score was highest in group $1 \quad(r=0.298$, $P<0.001)$.

3.4. Mean and Variance Analysis of RBG and FBG. Both RBG and FBG increased with the increasing Gensini score. The mean values of the RBG in groups $1-4$ were 9.08, 9.25, 10.19, and $10.83 \mathrm{mmol} / \mathrm{L}$, respectively. In addition, the mean values of the FBG were $7.29,7.64,8.20$, and $9.01 \mathrm{mmol} / \mathrm{L}$, respectively (Figure 1).

A one-way analysis of variance showed significant differences in RBG and FBG between the different Gensini score groups. The least significant difference (LSD) test showed that the RBG was significantly higher in groups 3 and 4 than in group $1 \quad(P=0.020$ and $P<0.001$, respectively). Furthermore, the FBG was significantly higher in groups 3 and 4 than in group $1(P=0.020$ and $P<0.001$, respectively). The FBG was higher in group 4 than in groups 2 and $3(P=0.001$ and $P=0.04)$ (Table 4$)$.

3.5. Multivariate Linear Regression Analysis. After adjusting for age, BMI, hypertension, CKD, smoking history, and serum lipid level, multiple linear regression analysis of the Gensini score as a continuous variable showed that sex, diabetes, eGFR, and FBG were independent risk factors related to the Gensini score $(r=9.770,8.366,-0.165$, and 1.540, respectively; and $P=0.015,0.043,0.044$, and 0.039 , respectively). RBG was not an independent risk factor related to the Gensini score $(r=1.145$ and $P=0.112)$ (Table 5).

Multiple logistic regression analysis showed that age and FBG were independent risk factors for the $37<$ Gensini
TABLE 1: Gensini score rule.

\begin{tabular}{lccc}
\hline $\begin{array}{l}\text { Degree of coronary artery } \\
\text { stenosis }\end{array}$ & Score & $\begin{array}{c}\text { Coronary lesion } \\
\text { site }\end{array}$ & Score \\
\hline$\leq 25 \%$ & 1 & LM & 5 \\
$26 \%-50 \%$ & 2 & Proximal LAD or & 2.5 \\
$51 \%-75 \%$ & 4 & Middle LAD & 1.5 \\
$76 \%-90 \%$ & 8 & Distal LAD & 1.0 \\
$91 \%-99 \%$ & 16 & Middle or distal & 1.0 \\
$100 \%$ & 32 & LAD & RCA \\
& & Subbranch & 0.5 \\
\hline
\end{tabular}

LM: left main coronary artery; LAD: left anterior descending; LCX: left circumflex coronary; RCA: right coronary artery.

score $\leq 60$ group $(P=0.001$ and $P=0.037$, respectively) compared to the Gensini score $\leq 37$ group. The eGFR and FBG were independent risk factors for the $60<$ Gensini score $\leq 88$ group $(P=0.014$ and $P=0.001$, respectively). Furthermore, the eGFR and FBG were independent risk factors for the Gensini score $>88$ group $(P=0.005$ and $P<0.001$, respectively). Neither diabetes nor RBG was an independent risk factor for Gensini score (Table 6).

\section{Discussion}

This study explored the correlation between blood glucose and the severity of coronary lesions in AMI patients. Our research showed that 265 of 958 AMI patients had admission hyperglycemia, which accounted for $27.66 \%$ of all AMI patients. Besides, 589 patients had elevated FBG, nearly 1.6 times as many as patients with normal fasting blood glucose. Statistical analysis showed that FBG rather than RBG is an independent risk factor for the Gensini score. Multivariate linear regression analysis showed the regression coefficient for FBG to be 1.6.

The incidence rate of diabetes is increasing worldwide due to population aging, poor dietary patterns, and lack of exercise [12]. Diabetes mellitus is an important risk factor for cardiovascular disease, with diabetes mellitus patients showing a 2-4-fold higher risk of cardiovascular disease than people with normal blood glucose levels. More than $70 \%$ of diabetes patients over 65 die from cardiovascular and cerebrovascular diseases [13]. One-third of patients who undergo coronary intervention are diabetic, and a quarter of patients treated with CABG have a history of diabetes [14]. Coronary lesions tend to have greater inflammatory cell infiltration and more diffuse lesions in diabetic patients [15]. Diabetes is a risk factor for AMI, and diabetic patients with AMI have more severe lesions and an increased risk of poor short-term and long-term prognoses [16].

Stress hyperglycemia refers to transient hyperglycemia that occurs during the course of disease. The ADA defines stress hyperglycemia as an FBG level $>6.9 \mathrm{mmol} / \mathrm{L}$ or $\mathrm{RBG}$ level $>11.1 \mathrm{mmol} / \mathrm{L}$ without diabetes [10]. Stress hyperglycemia is closely related to mortality in AMI patients. In a meta-analysis involving $1856 \mathrm{AMI}$ patients [8], the relative risk of in-hospital death in nondiabetic patients with stress hyperglycemia was 3.9-fold higher than that in patients with 
TABLE 2: Comparison of baseline data, hematological indexes, coronary angiography data, and Gensini score between the hyperglycemia group and nonhyperglycemia group.

\begin{tabular}{|c|c|c|c|}
\hline Variables & Hyperglycemia group $(n=265)$ & Nonhyperglycemia group $(n=693)$ & $P$ value \\
\hline Sex (man) & 193 & 551 & $0.026^{*}$ \\
\hline Age & $64.08 \pm 12.94$ & $61.64 \pm 13.47$ & $0.019^{*}$ \\
\hline Height $(\mathrm{cm})$ & $166.71 \pm 7.44$ & $166.78 \pm 7.54$ & 0.92 \\
\hline Weight (kg) & $68.55 \pm 12.18$ & $68.76 \pm 11.38$ & 0.844 \\
\hline $\operatorname{BMI}\left(\mathrm{kg} / \mathrm{m}^{2}\right)$ & $24.93 \pm 3.87$ & $24.44 \pm 3.53$ & 0.132 \\
\hline SBP (mmHg) & $124.80 \pm 24.80$ & $126.52 \pm 20.83$ & 0.315 \\
\hline DBP (mmHg) & $76.14 \pm 16.29$ & $76.98 \pm 14.32$ & 0.472 \\
\hline Hypertension & 167 & 384 & $0.033^{*}$ \\
\hline Diabetes & 174 & 88 & $<0.001^{*}$ \\
\hline Previous MI & 12 & 10 & $0.013^{*}$ \\
\hline Cerebral infarction & 37 & 65 & $0.040^{*}$ \\
\hline CKD & 30 & 49 & $0.032^{*}$ \\
\hline Smoking & 119 & 380 & $0.006^{*}$ \\
\hline \multicolumn{4}{|l|}{ Killip grade } \\
\hline I grade & 158 & 502 & $<0.001^{*}$ \\
\hline II grade & 42 & 112 & 0.906 \\
\hline III grade & 2 & 9 & 0.480 \\
\hline IV grade & 63 & 70 & $<0.001^{*}$ \\
\hline TNI $\mathrm{P}_{50}\left(\mathrm{P}_{25}-\mathrm{P}_{75}\right)(\mathrm{ng} / \mathrm{ml})$ & $17.9(3.2-25)$ & $14(3.53-25)$ & 0.286 \\
\hline Myo $\mathrm{P}_{50}\left(\mathrm{P}_{25}-\mathrm{P}_{75}\right)(\mathrm{ng} / \mathrm{ml})$ & $500(241-900)$ & $500(239-900)$ & 0.096 \\
\hline CK-MB $\mathrm{P}_{50}\left(\mathrm{P}_{25}-\mathrm{P}_{75}\right)(\mathrm{ng} / \mathrm{ml})$ & $80(26.9-292)$ & $80(44-303.25)$ & 0.406 \\
\hline $\mathrm{BNP}_{50}\left(\mathrm{P}_{25}-\mathrm{P}_{75}\right)(\mathrm{pg} / \mathrm{ml})$ & $216.5(55.9-862)$ & $134(43.3-393.25)$ & $0.002^{*}$ \\
\hline WBC $\left({ }^{*} 10^{9} / \mathrm{L}\right)$ & $12.46 \pm 4.61$ & $11.19 \pm 3.68$ & $<0.001^{*}$ \\
\hline $\mathrm{Hb}(\mathrm{g} / \mathrm{L})$ & $133.21 \pm 23.15$ & $135.99 \pm 20.71$ & 0.098 \\
\hline $\operatorname{PLT}\left({ }^{*} 10^{9} / \mathrm{L}\right)$ & $207.48 \pm 66.48$ & $204.68 \pm 60.07$ & 0.564 \\
\hline $\mathrm{RBG}(\mathrm{mmol} / \mathrm{L})$ & $16.34 \pm 4.85$ & $7.35 \pm 1.62$ & $<0.001^{*}$ \\
\hline $\operatorname{ALT~} \mathrm{P}_{50}\left(\mathrm{P}_{25}-\mathrm{P}_{75}\right)(\mathrm{IU} / \mathrm{L})$ & $53(34-82)$ & $44(30-68.75)$ & $0.001^{*}$ \\
\hline AST $\mathrm{P}_{50}\left(\mathrm{P}_{25}-\mathrm{P}_{75}\right)(\mathrm{IU} / \mathrm{L})$ & $174.5(79-323)$ & $157.5(76.75-262.25)$ & 0.073 \\
\hline $\operatorname{Scr} \mathrm{P}_{50}\left(\mathrm{P}_{25}-\mathrm{P}_{75}\right)(\mu \mathrm{mol} / \mathrm{L})$ & $81(71-105.5)$ & $80.5(69-92)$ & 0.1 \\
\hline eGFR $P_{50}\left(P_{25}-P_{75}\right)\left(\mathrm{ml} / \mathrm{min} / 1.73 \mathrm{~m}^{2}\right)$ & $77.18(54.95-96.24)$ & $82.37(68.89-97.27)$ & $0.022^{*}$ \\
\hline $\mathrm{UA}(\mu \mathrm{mol} / \mathrm{L})$ & $364.18 \pm 143.79$ & $366.75 \pm 115.43$ & 0.079 \\
\hline FBG $(\mathrm{mmoL} / \mathrm{L})$ & $12.00 \pm 5.47$ & $6.45 \pm 2.02$ & $<0.001^{*}$ \\
\hline TG $(\mathrm{mmoL} / \mathrm{L})$ & $2.12 \pm 2.12$ & $1.67 \pm 1.15$ & $0.004^{*}$ \\
\hline $\mathrm{TC}(\mathrm{mmoL} / \mathrm{L})$ & $4.27 \pm 1.34$ & $4.48 \pm 1.35$ & $0.044^{*}$ \\
\hline HDL-c (mmoL/L) & $1.02 \pm 0.25$ & $1.13 \pm 0.41$ & $<0.001^{*}$ \\
\hline $\mathrm{LDL}-\mathrm{c}(\mathrm{mmoL} / \mathrm{L})$ & $2.62 \pm 0.99$ & $2.80 \pm 0.86$ & $0.017^{*}$ \\
\hline LVEF (\%) & $0.54 \pm 0.12$ & $0.58 \pm 0.11$ & $<0.0010^{*}$ \\
\hline Aspirin & 263 & 681 & 0.26 \\
\hline Clopidogrel & 21 & 95 & $0.014^{*}$ \\
\hline Ticagrelor & 244 & 598 & $0.014^{*}$ \\
\hline ACEI/ARB & 112 & 370 & $0.002^{*}$ \\
\hline$\beta$-blocker & 214 & 580 & 0.28 \\
\hline Statin & 265 & 688 & 0.166 \\
\hline $\mathrm{CCB}$ & 16 & 44 & 0.338 \\
\hline Furosemide & 72 & 177 & 0.424 \\
\hline Antisterone & 70 & 179 & 0.261 \\
\hline IABP & 28 & 35 & $0.002^{*}$ \\
\hline CABG & 5 & 8 & 0.381 \\
\hline \multicolumn{4}{|l|}{ Coronary lesions } \\
\hline Single-vessel lesion & 44 & 192 & $<0.001^{*}$ \\
\hline Double-vessel lesions & 95 & 191 & $0.012^{*}$ \\
\hline Triple-vessel lesions & 126 & 310 & 0.434 \\
\hline \multicolumn{4}{|l|}{ Number of stents } \\
\hline Single stent & 167 & 415 & 0.374 \\
\hline Two or more stents & 44 & 133 & 0.356 \\
\hline Left main lesion & 37 & 62 & $0.023^{*}$ \\
\hline Gensini score $\mathrm{P}_{50}\left(\mathrm{P}_{25}-\mathrm{P}_{75}\right)$ & $71.5(41-92)$ & $58(36-87)$ & $0.006^{*}$ \\
\hline
\end{tabular}

BMI: body mass index; SBP: systolic blood pressure; DBP: diastolic blood pressure; CKD: chronic kidney diseases; TNI: troponin; Myo: myohemoglobin; CKMB: creatine kinase-MB; BNP: brain natriuretic peptide; WBC: white blood cell; Hb: hemoglobin; PLT: platelet; RBG: random blood glucose; ALT: alanine transaminase; AST: Aspartate transaminase; Scr: serum creatinine; eGFR: estimated glomerular filtration rate; UA: uric acid; FBG: fasting blood glucose; TG: triglyceride; TC: total cholesterol; HDL-c: high-density lipoprotein-cholesterol; LDL-c: low-density lipoprotein-cholesterol; LVEF: left ventricular ejection fraction; CCB: calcium channel blocker; IABP: intra-aortic balloon pump; CABG: coronary artery bypass grafting. 
TABLE 3: Comparison of baseline data, hematological parameters, coronary angiography data, and Gensini score between the FBG elevated group and the non-FBG elevated group.

\begin{tabular}{|c|c|c|c|}
\hline Variables & FBG elevated group $(n=589)$ & Non-FBG elevated group $(n=369)$ & $P$ value \\
\hline Sex (man) & 433 & 303 & $0.002^{*}$ \\
\hline Age & $62.91 \pm 13.01$ & $61.72 \pm 13.84$ & 0.185 \\
\hline Height $(\mathrm{cm})$ & $166.77 \pm 7.64$ & $166.70 \pm 7.20$ & 0.087 \\
\hline Weight (kg) & $69.02 \pm 12.20$ & $68.15 \pm 11.73$ & 0.319 \\
\hline $\operatorname{BMI}\left(\mathrm{kg} / \mathrm{m}^{2}\right)$ & $24.77 \pm 3.70$ & $24.09 \pm 3.71$ & $0.012^{*}$ \\
\hline SBP (mmHg) & $127.08 \pm 23.18$ & $125.34 \pm 19.14$ & 0.235 \\
\hline $\mathrm{DBP}(\mathrm{mmHg})$ & $76.89 \pm 15.05$ & $76.44 \pm 13.84$ & 0.467 \\
\hline Hypertension & 349 & 202 & 0.169 \\
\hline Diabetes & 242 & 20 & $<0.001^{*}$ \\
\hline Previous MI & 15 & 7 & 0.514 \\
\hline Cerebral infarction & 86 & 16 & $<0.001^{*}$ \\
\hline CKD & 54 & 25 & 0.190 \\
\hline Smoking & 283 & 216 & $0.002^{*}$ \\
\hline \multicolumn{4}{|l|}{ Killip grade } \\
\hline I grade & 367 & 293 & $<0.001^{*}$ \\
\hline II grade & 103 & 51 & 0.133 \\
\hline III grade & 9 & 2 & 0.163 \\
\hline IV grade & 99 & 34 & $<0.001^{*}$ \\
\hline TNI $\mathrm{P}_{50}\left(\mathrm{P}_{25}-\mathrm{P}_{75}\right)(\mathrm{ng} / \mathrm{ml})$ & $17(3.98-25)$ & $11(2.3-25)$ & $0.002^{*}$ \\
\hline Myo $\mathrm{P}_{50}\left(\mathrm{P}_{25}-\mathrm{P}_{75}\right)(\mathrm{ng} / \mathrm{ml})$ & $500(219.75-900)$ & $500(206.25-900)$ & $0.016^{*}$ \\
\hline CK-MB $\mathrm{P}_{50}\left(\mathrm{P}_{25}-\mathrm{P}_{75}\right)(\mathrm{ng} / \mathrm{ml})$ & $80(36.45-297)$ & $80(37.5-246.5)$ & 0.260 \\
\hline BNP $\mathrm{P}_{50}\left(\mathrm{P}_{25}-\mathrm{P}_{75}\right)(\mathrm{pg} / \mathrm{ml})$ & $173(49.75-587.5)$ & $112(41.45-347.5)$ & $0.001^{*}$ \\
\hline WBC $\left({ }^{*} 10^{9} / \mathrm{L}\right)$ & $12.29 \pm 4.45$ & $10.47 \pm 2.94$ & $<0.001^{*}$ \\
\hline $\mathrm{Hb}(\mathrm{g} / \mathrm{L})$ & $134.5 \pm 23.11$ & $135.99 \pm 20.71$ & 0.066 \\
\hline $\operatorname{PLT}\left({ }^{*} 10^{9} / \mathrm{L}\right)$ & $209.87 \pm 63.42$ & $201.7 \pm 58.36$ & 0.051 \\
\hline $\mathrm{RBG}(\mathrm{mmol} / \mathrm{L})$ & $11.25 \pm 5.21$ & $7.22 \pm 2.35$ & $<0.001^{*}$ \\
\hline $\operatorname{ALT~} \mathrm{P}_{50}\left(\mathrm{P}_{25}-\mathrm{P}_{75}\right)(\mathrm{IU} / \mathrm{L})$ & $48(33-79)$ & $39(29-59)$ & $<0.001^{*}$ \\
\hline AST $\mathrm{P}_{50}\left(\mathrm{P}_{25}-\mathrm{P}_{75}\right)(\mathrm{IU} / \mathrm{L})$ & $145(60-219)$ & $171(89-309)$ & $<0.001^{*}$ \\
\hline Scr $\mathrm{P}_{50}\left(\mathrm{P}_{25}-\mathrm{P}_{75}\right)(\mu \mathrm{mol} / \mathrm{L})$ & $81(67-98)$ & $80(72-91)$ & 0.862 \\
\hline eGFR $P_{50}\left(P_{25}-P_{75}\right)\left(\mathrm{ml} / \mathrm{min} / 1.73 \mathrm{~m}^{2}\right)$ & $80.91(62.22-97.88)$ & $81.56(69.16-96.16)$ & 0.704 \\
\hline $\mathrm{UA}(\mu \mathrm{mol} / \mathrm{L})$ & $376.41 \pm 143.79$ & $365.35 \pm 122.55$ & 0.152 \\
\hline $\mathrm{FBG}(\mathrm{mmoL} / \mathrm{L})$ & $9.79 \pm 4.67$ & $5.22 \pm 0.52$ & $<0.001^{*}$ \\
\hline TG $(\mathrm{mmoL} / \mathrm{L})$ & $1.83 \pm 1.66$ & $1.64 \pm 0.98$ & 0.051 \\
\hline $\mathrm{TC}(\mathrm{mmoL} / \mathrm{L})$ & $4.46 \pm 1.34$ & $4.42 \pm 0.98$ & 0.667 \\
\hline HDL-c (mmoL/L) & $1.12 \pm 0.44$ & $1.07 \pm 0.30$ & $0.046^{*}$ \\
\hline LDL-c (mmoL/L) & $2.77 \pm 0.99$ & $2.80 \pm 0.82$ & 0.682 \\
\hline $\operatorname{LVEF}(\%)$ & $0.53 \pm 0.38$ & $0.68 \pm 0.34$ & $<0.001^{*}$ \\
\hline Aspirin & 581 & 364 & 0.997 \\
\hline Clopidogrel & 61 & 55 & $0.028^{*}$ \\
\hline Ticagrelor & 528 & 314 & $0.028^{*}$ \\
\hline ACEI/ARB & 277 & 205 & $0.01^{*}$ \\
\hline$\beta$-blocker & 494 & 300 & 0.304 \\
\hline Statin & 586 & 367 & 0.946 \\
\hline $\mathrm{CCB}$ & 30 & 30 & 0.059 \\
\hline Furosemide & 174 & 75 & $0.002^{*}$ \\
\hline Antisterone & 172 & 77 & $0.004^{*}$ \\
\hline IABP & 51 & 12 & $0.001^{*}$ \\
\hline CABG & 10 & 3 & 0.249 \\
\hline \multicolumn{4}{|l|}{ Coronary lesions } \\
\hline Single-vessel lesion & 101 & 135 & $<0.001^{*}$ \\
\hline Double-vessel lesions & 171 & 115 & 0.483 \\
\hline Triple-vessel lesions & 284 & 152 & $0.034^{*}$ \\
\hline \multicolumn{4}{|l|}{ Number of stents } \\
\hline Single stent & 340 & 242 & $0.015^{*}$ \\
\hline Two or more stents & 124 & 53 & $0.009^{*}$ \\
\hline Left main lesion & 75 & 24 & $0.002^{*}$ \\
\hline Gensini score $\mathrm{P}_{50}\left(\mathrm{P}_{25}-\mathrm{P}_{75}\right)$ & $65(42-92)$ & $52(32-82)$ & $<0.001^{*}$ \\
\hline
\end{tabular}

SBP: systolic blood pressure; DBP: diastolic blood pressure; CKD: chronic kidney diseases; TNI: troponin; Myo: myohemoglobin; CK-MB: creatine kinaseMB; BNP: brain natriuretic peptide; WBC: white blood cell; Hb: hemoglobin; PLT: platelet; RBG: random blood glucose; ALT: alanine transaminase; AST: aspartate transaminase; Scr: serum creatinine; eGFR: estimated glomerular filtration rate; UA: uric acid; FBG: fasting blood glucose; TG: triglyceride; TC: total cholesterol; HDL-c: high-density lipoprotein-cholesterol; LDL-c: low-density lipoprotein-cholesterol; LVEF: left ventricular ejection fraction; CCB: calcium channel blocker; IABP: intra-aortic balloon pump; CABG: coronary artery bypass grafting. 


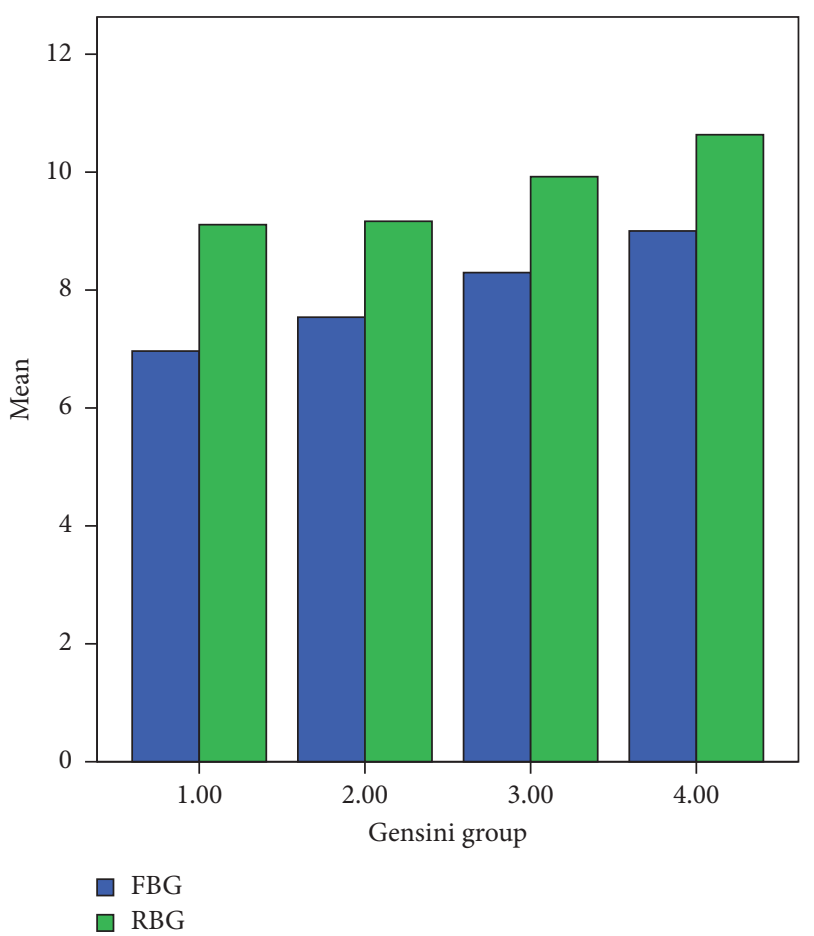

Figure 1: RBG and FBG mean distributions in different Gensini score groups.

TABle 4: RBG and FBG in different Gensini score groups by the LSD method.

\begin{tabular}{cccccccccc}
\hline & & \multicolumn{4}{c}{ RBG } & & \multicolumn{2}{c}{ FBG } \\
Group & Group & Mean difference & \multicolumn{2}{c}{$95 \%$ CI } & & \multicolumn{2}{c}{$95 \%$ CI } \\
& & & Lower limit & Upper limit & $P$ value & Mean difference & Lower limit & Upper limit & $P$ value \\
\hline 1 & 2 & -0.17 & -0.13 & 0.79 & 0.733 & -0.35 & -1.12 & 0.42 & 0.378 \\
& 3 & -1.11 & -2.05 & -0.17 & $0.020^{*}$ & -0.91 & -1.67 & -0.14 & $0.020^{*}$ \\
& 4 & -1.75 & -2.71 & -0.80 & $<0.001^{*}$ & -1.72 & -2.5 & -0.94 & $<0.001^{*}$ \\
2 & 3 & -0.94 & -1.89 & 0.01 & 0.051 & -0.56 & -1.33 & 0.21 & 0.152 \\
& 4 & -1.59 & -2.59 & -0.62 & $0.001^{*}$ & -1.37 & -2.15 & -0.59 & $0.001^{*}$ \\
3 & 4 & -0.64 & -1.59 & 0.30 & 0.183 & -0.81 & -1.59 & -0.04 & $0.04^{*}$ \\
\hline
\end{tabular}

RBG: random blood glucose; FBG: fasting blood glucose. ${ }^{*} P<0.05$.

TABLE 5: Multivariate linear regression analysis for Gensini score as a continuous variable.

\begin{tabular}{lcc}
\hline Variables & $\beta$ & $P$ value \\
\hline Sex & 9.770 & $0.015^{*}$ \\
Age & 0.125 & 0.098 \\
BMI & 0.05 & 0.793 \\
Hypertension & 1.147 & 0.701 \\
Diabetes & 8.366 & $0.043^{*}$ \\
CKD & 0.508 & 0.936 \\
Smoking & 2.453 & 0.467 \\
eGFR & -0.165 & $0.044^{*}$ \\
FBG & 1.540 & $0.039^{*}$ \\
RBG & 1.145 & 0.112 \\
TG & 0.409 & 0.138 \\
TC & 2.254 & 0.252 \\
HDL-c & 0.465 & 0.372 \\
LDL-c & -0.503 & 0.636 \\
\hline
\end{tabular}

CKD: chronic kidney diseases; eGFR: estimated glomerular filtration rate; RBG: random blood glucose; FBG: fasting blood glucose; TG: triglyceride; TC: total cholesterol; HDL-c: high-density lipoprotein-cholesterol; LDL-c: low-density lipoprotein-cholesterol. normal blood glucose levels (95\% CI $=2.9-5.4)$, and diabetic patients with stress hyperglycemia were 1.7-fold more likely to die in hospital than those with normal blood glucose levels (95\% CI $=1.2-2.4)$. Approximately half of all patients with ST-segment elevation MI (STEMI) are admitted with stress hyperglycemia [17], which can increase the MI area, severity of CAD, and mortality, leading to other adverse outcomes $[18,19]$; decreased left ventricular systolic function may also be found [20]. The IABP-SHOCK II study showed that, in AMI patients with cardiogenic shock, RBG was an independent predictor of 30-day and 1-year mortality after MI. The 30-day mortality rates of patients with an RBG level $\geq 11.5 \mathrm{mmol} / \mathrm{L}$ and an RBG level $<11.5 \mathrm{mmol} / \mathrm{L}$ were $47.7 \%$ vs. $36.5 \%$, respectively $(P=0.004)$, and the corresponding 1 year mortality rates were $57.7 \%$ vs. $47.1 \%$, respectively $(P=0.011)[21]$. Among acute coronary syndrome patients, patients with stress hyperglycemia had a higher Gensini score, an increased incidence of 6-month major adverse cardiac events and an increased incidence of in-hospital arrhythmia [22]. RBG interferes with blood flow in the 
TABle 6: Multivariate logistic regression analysis for the Gensini score as a hierarchical variable.

\begin{tabular}{lcccc}
\hline Gensini score group & Variables & OR & $95 \%$ CI & $P$ value \\
\hline Gensini score $\leq 37$ & & & & \\
& Sex & 1.20 & $0.729-1.974$ & 0.473 \\
& Age & 1.031 & $1.013-1.050$ & $0.001^{*}$ \\
$37<$ Gensini score $\leq 60$ & Diabetes & 1.001 & $0.513-1.812$ & 0.997 \\
& eGFR & 1.006 & $0.000-1.014$ & 0.090 \\
& RBG & 0.941 & $0.878-1.009$ & 0.087 \\
& FBG & 1.111 & $1.006-1.226$ & $0.037^{*}$ \\
& Sex & 1.233 & $0.746-2.039$ & 0.414 \\
& Age & 0.99 & $0.973-1.007$ & 0.260 \\
$60<$ Gensini score $\leq 88$ & Diabetes & 0.949 & $0.573-1.679$ & 0.858 \\
& eGFR & 0.99 & $0.983-0.998$ & $0.014^{*}$ \\
& RBG & 0.959 & $0.901-1.002$ & 0.199 \\
& FBG & 1.067 & $1.006-1.279$ & $0.001^{*}$ \\
& Sex & 0.688 & $0.400-1.182$ & 0.176 \\
& Age & 1.018 & $0.999-1.037$ & 0.057 \\
Gensini score $>88$ & Diabetes & 0.615 & $0.337-1.120$ & 0.112 \\
& eGFR & 0.988 & $0.980-0.996$ & $0.005^{*}$ \\
& RBG & 0.986 & $0.926-1.050$ & 0.667 \\
& FBG & 1.202 & $1.097-1.316$ & $<0.001^{*}$ \\
\hline
\end{tabular}

eGFR: estimated glomerular filtration rate; RBG: random blood glucose; FBG: fasting blood glucose. ${ }^{*} P<0.05$.

culprit vessel and is linearly correlated with the TIMI blood flow index [23]. Some studies have indicated that RBG on admission is closely related to mortality in nondiabetic patients [24].

AMI is a life-threatening acute disease, in the acute phase of the disease, the accumulation of oxidative stress, cytokines, catecholamines, cortisol, and inflammatory markers results in a decrease in gluconeogenesis and an acceleration of glycogen decomposition leading to hyperglycemia [25]. Hyperglycemia is also associated with endothelial barrier injury, activation of NF-kappa-b, impaired endotheliumdependent vascular diastolic function, and the overexpression of adhesion molecules such as intercellular adhesion molecules, vascular cell adhesion molecules, and e-selectin [26, 27]. Catecholamines, cortisol, and other hormones are also increased, leading to increased glucose utilization, which is conducive to cell hibernation and prevents further apoptosis in AMI patients [28]. Previous animal studies demonstrated that SGLT2 inhibition reduced the mortality of diabetic rats after MI through cardiac energy metabolism and the protective modification of antioxidant proteins, suggesting that hyperglycemia is related to a disturbance in energy metabolism and oxidative stress during AMI [29]. In patients with abnormal glucose tolerance and diabetes mellitus, catalase, superoxide dismutase, glutathione $(\mathrm{GSH}), \mathrm{GSH}$ reductase, and GSH peroxidase were negatively correlated with the Gensini score, suggesting that oxidative stress is involved in the mechanism underlying the aggravation of CAD by hyperglycemia [30].

IFG refers to a fasting glucose level of $6.1-7.0 \mathrm{mmol} / \mathrm{L}$. In a 5-year follow-up of newly diagnosed IFG patients at the Singapore National Hospital, 20 (0.9\%) of the 2295 IFG patients developed AMI, which was significantly higher than the incidence in the normal blood glucose group [31]. In a prospective study, Uppalakal and Karanayil [32] reported a
$40 \%$ incidence of metabolic syndrome in STEMI patients, 93\% of whom had an elevated FBG level (FBG > $100 \mathrm{mg}$ ). Elevated FBG is an independent risk factor for CAD (odds ratio $(\mathrm{OR})=2.238,95 \% \mathrm{CI}=1.111-4.508, P=0.024)$; reducing FBG is beneficial for preventing the occurrence and reducing the incidence of CAD [33]. Elevated FBG is associated with the severity of CAD and is correlated with the Gensini score [34]. In a study of 1028 patients who underwent coronary angiography, patients with an elevated FBG level had more severe coronary artery lesions and higher Gensini scores $(P<0.001)$. A meta-analysis [35] also indicated that IFG was an independent risk factor for cardiovascular disease $(\mathrm{OR}=1.12-1.37)$. These results are consistent with those of the present study. However, some authors have reported the opposite findings; a follow-up study of 11338 patients indicated no significant difference in the severity of CAD and the incidence of composite cardiovascular endpoint events in patients with prediabetes compared to the normal population [36]. The FBG and postprandial blood glucose (PBG) were positively correlated with the Gensini score in 1852 patients who underwent coronary angiography $(r=0.09, P<0.01$; and $r=0.20$, $P<0.01$, respectively), whereas regression analysis showed that FBG rather than PBG was independently associated with Gensini score.

This study has several limitations. Firstly, this was a single-center study with a small sample size. A multicenter study with a larger sample size is needed to further evaluate the correlations between RBG, FBG, and Gensini score and to determine the predictive value for short-term and longterm adverse prognoses. Secondly, this study did not explore the correlation between PBG and Gensini score. Thirdly, we did not report the exact $\mathrm{HbA} 1 \mathrm{c}$ value. Finally, this study did not clarify whether interventions for hyperglycemia can reverse its adverse effects.

\section{Conclusions}

In conclusion, we should pay more attention to FBG, and timely intervention for hyperglycemia, by using insulin or other glucose-lowering medication, may bring more benefit for patients with myocardial infarction.

\section{Data Availability}

The data that support the findings of this study are available from the corresponding author upon reasonable request.

\section{Conflicts of Interest}

The authors certify that they have no affiliations with or involvement in any organization or entity with any financial interest or nonfinancial interest in the subject matter or materials discussed in this manuscript.

\section{Acknowledgments}

The research was funded by the National Natural Science Foundation of China (grant no. 81600227). 


\section{References}

[1] W. B. Kannel and D. L. McGee, "Diabetes and cardiovasculardisease-Framingham-study," Journal of the American Medical Association, vol. 241, no. 19, pp. 2035-2038, 1979.

[2] S. M. Haffner, S. Lehto, T. Ronnemaa, K. Pyorala, and M. Laakso, "Mortality from coronary heart disease in subjects with type 2 diabetes and in nondiabetic subjects with and without prior myocardial infarction," New England Journal of Medicine, vol. 339, no. 4, pp. 229-234, 1998.

[3] R. Naito and K. Miyauchi, "Coronary artery disease and type 2 diabetes mellitus current treatment strategies and future perspective," International Heart Journal, vol. 58, no. 4, pp. 475-480, 2017.

[4] S. Ertek, A. F. Cicero, M. Cesur et al., "The severity of coronary atherosclerosis in diabetic and non-diabetic metabolic syndrome patients diagnosed according to different criteria and undergoing elective angiography," Acta Diabetologica, vol. 48, no. 1, pp. 21-27, 2011.

[5] S. Yusuf, S. Hawken, S. Ounpuu et al., "Effect of potentially modifiable risk factors associated with myocardial infarction in 52 countries (the INTERHEART study): case-control study," The Lancet, vol. 364, no. 9438, pp. 937-952, 2004.

[6] DECODE Study Group and European Diabetes Epidemiology Group, "Is the current definition for diabetes relevant to mortality risk from all causes and cardiovascular and noncardiovascular diseases?," Diabetes Care, vol. 26, no. 3, pp. 688-696, 2003.

[7] S. Gurudevan, P. Garg, S. Malik et al., "Impaired fasting glucose is associated with increased severity of subclinical coronary artery disease compared to patients with diabetes and normal fasting glucose: evaluation by coronary computed tomographic angiography," BMJ Open, vol. 6, no. 8, Article ID e005148, 2016.

[8] S. E. Capes, D. Hunt, K. Malmberg, and H. C. Gerstein, "Stress hyperglycaemia and increased risk of death after myocardial infarction in patients with and without diabetes: a systematic overview," The Lancet, vol. 355, no. 9206, pp. 773-778, 2000.

[9] R. C. Turner, R. R. Holman, C. A. Cull et al., "Intensive bloodglucose control with sulphonylureas or insulin compared with conventional treatment and risk of complications in patients with type 2 diabetes (UKPDS 33)," The Lancet, vol. 352, no. 9131, pp. 837-853, 1998.

[10] K. M. Dungan, S. S. Braithwaite, and J. C. Preiser, "Stress hyperglycaemia," The Lancet, vol. 373, no. 9677, pp. 1798$1807,2009$.

[11] G. G. Gensini, "A more meaningful scoring system for determining the severity of coronary heart disease," American Journal of Cardiology, vol. 51, no. 3, p. 606, 1983.

[12] G. Danaei, M. M. Finucane, Y. Lu et al., "National, regional, and global trends in fasting plasma glucose and diabetes prevalence since 1980: systematic analysis of health examination surveys and epidemiological studies with 370 countryyears and 2.7 million participants," The Lancet, vol. 378, no. 9785, pp. 31-40, 2011.

[13] A. S. Go, D. Mozaffarian, V. L Roger et al., "Heart disease and stroke statistics--2014 update: a report from the American Heart Association," Circulation, vol. 129, no. 3, pp. e28-E292, 2014.

[14] C. Berry, J. C. Tardif, and M. G. Bourassa, "Coronary heart disease in patients with diabetes: part II: recent advances in coronary revascularization," Journal of the American College of Cardiology, vol. 49, no. 6, pp. 643-656, 2007.
[15] R. Virmani, A. P. Burke, and F. Kolodgie, "Morphological characteristics of coronary atherosclerosis in diabetes mellitus," Canadian Journal of Cardiology, vol. 22, pp. 81B-4B, 2006.

[16] S. M. Donahoe, G. C. Stewart, C. H McCabe et al., "Diabetes and mortality following acute coronary syndromes," Journal of the American Medical Association, vol. 298, no. 7, pp. 765-775, 2007.

[17] M. Kosiborod and D. K. McGuire, "Glucose-lowering targets for patients with cardiovascular disease: focus on inpatient management of patients with acute coronary syndromes," Circulation, vol. 122, no. 25, pp. 2736-2744, 2010.

[18] J. R. Timmer, M. Hoekstra, M. W. N. Nijsten et al., "Prognostic value of admission glycosylated hemoglobin and glucose in nondiabetic patients with ST-segment-elevation myocardial infarction treated with percutaneous coronary intervention," Circulation, vol. 124, no. 6, pp. 704-711, 2011.

[19] M. Kosiborod, S. S. Rathore, S. E. Inzucchi et al., "Admission glucose and mortality in elderly patients hospitalized with acute myocardial infarction-implications for patients with and without recognized diabetes," Circulation, vol. 111, no. 23, pp. 3078-3086, 2005.

[20] M. Ishihara, I. Inoue, T. Kawagoe et al., "Impact of acute hyperglycemia on left ventricular function after reperfusion therapy in patients with a first anterior wall acute myocardial infarction," American Heart Journal, vol. 146, no. 4, pp. 674-678, 2003.

[21] A. Abdin, J. Pöss, G. Fuernau et al., "Revision: prognostic impact of baseline glucose levels in acute myocardial infarction complicated by cardiogenic shock-a substudy of the IABP-SHOCK II-trial," Clinical Research in Cardiology, vol. 107, no. 6, pp. 517-523, 2018.

[22] H. Ayhan, T. Durmaz, T. Keleş et al., "The relationship between acute coronary syndrome and stress hyperglycemia," Experimental and Clinical Endocrinology \& Diabetes, vol. 122, no. 04 , pp. 222-226, 2014.

[23] M. Pepe, D. Zanna, A. Cafaro et al., "Role of plasma glucose level on myocardial perfusion in ST-segment elevation myocardial infarction patients," Journal of Diabetes and Its Complications, vol. 32, no. 8, pp. 764-769, 2018.

[24] S. Kim, S. J. Na, T. K. Park et al., "Prognostic value of admission blood glucose level in critically ill patients admitted to cardiac intensive care unit according to the presence or absence of diabetes mellitus," Journal of Korean Medical Science, vol. 34, no. 9, p. 9, 2019.

[25] A. Norhammar, A. Tenerz, G. Nilsson et al., "Glucose metabolism in patients with acute myocardial infarction and no previous diagnosis of diabetes mellitus: a prospective study," The Lancet, vol. 359, no. 9324, pp. 2140-2144, 2002.

[26] R. Piga, Y. Naito, S. Kokura, O. Handa, and T. Yoshikawa, "Short-term high glucose exposure induces monocyte-endothelial cells adhesion and transmigration by increasing VCAM-1 and MCP-1 expression in human aortic endothelial cells," Atherosclerosis, vol. 193, no. 2, pp. 328-334, 2007.

[27] K. Naruse, C. Rask-Madsen, N. Takahara et al., "Activation of vascular protein kinase $\mathrm{C}$ - inhibits akt-dependent endothelial nitric oxide synthase function in obesity-associated insulin resistance," Diabetes, vol. 55, no. 3, pp. 691-698, 2006.

[28] E. F. Oliver and L. H. Opie, "Effects of glucose and fatty acids on myocardial ischaemia and arrhythmias," The Lancet, vol. 343, no. 8890, pp. 155-158, 1994.

[29] H. Oshima, T. Miki, A. Kuno et al., "Empagliflozin, an SGLT2 inhibitor, reduced the mortality rate after acute myocardial infarction with modification of cardiac metabolomes and 
antioxidants in diabetic rats," Journal of Pharmacology and Experimental Therapeutics, vol. 368, no. 3, pp. 524-534, 2019.

[30] W. Liang, Y.-J. Zhao, H. Yang, and L.-H. Shen, "Effects of antioxidant system on coronary artery lesions in patients with abnormal glucose metabolism," Aging Clinical and Experimental Research, vol. 29, no. 2, pp. 141-146, 2017.

[31] D. B. Chia, L. Y. Wong, D. Y. K. Liu, and M. P. H. S. Toh, "Predictive factors of developing type 2 diabetes mellitus, acute myocardial infarction and stroke in a cohort with impaired fasting glucose in Singapore," Diabetes Research and Clinical Practice, vol. 132, pp. 59-67, 2017.

[32] B. Uppalakal and L. S. Karanayil, "Incidence of metabolic syndrome in patients admitted to medical wards with ST elevation myocardial infarction," Journal of Clinical and Diagnostic Research, vol. 11, no. 3, pp. OC17-OC20, 2017.

[33] W. Zidi, M. Allal-Elasmi, Y. Zayani et al., "Metabolic syndrome, independent predictor for coronary artery disease," Clinical Laboratory, vol. 61, no. 10, pp. 1545-1552, 2015.

[34] X. Y. Zhang, L. N. Dong, Q. Wang, and X. M. Xie, "The relationship between fasting plasma glucose and MPO in patients with acute coronary syndrome," BMC Cardiovascular Disorders, vol. 15, 2015.

[35] E. S. Ford, G. Zhao, and C. Li, "Pre-diabetes and the risk for cardiovascular disease a systematic review of the evidence," Journal of the American College of Cardiology, vol. 55, no. 13, pp. 1310-1317, 2010.

[36] H.-H. Liu, Y.-X. Cao, S. Li et al., "Impacts of prediabetes mellitus alone or plus hypertension on the coronary severity and cardiovascular outcomes," Hypertension, vol. 71, no. 6, pp. 1039-1046, 2018. 


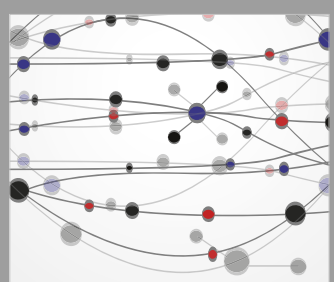

The Scientific World Journal
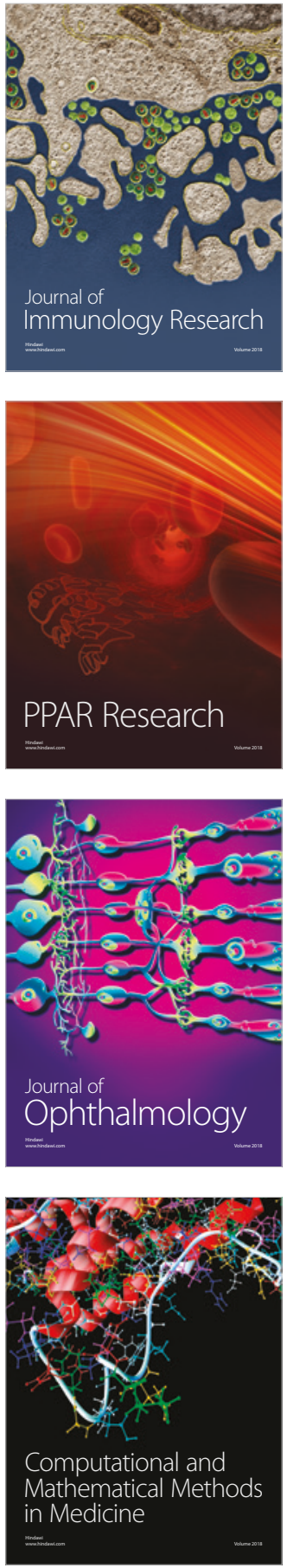

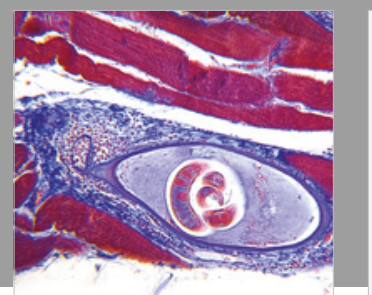

Gastroenterology Research and Practice

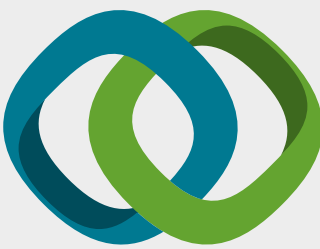

\section{Hindawi}

Submit your manuscripts at

www.hindawi.com
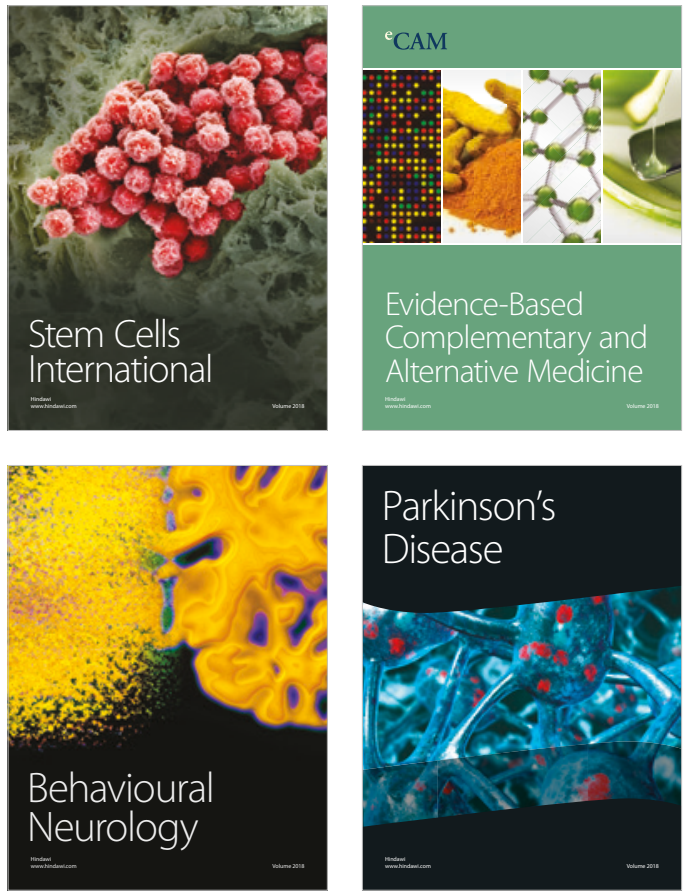

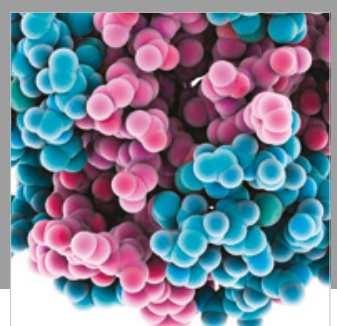

ournal of

Diabetes Research

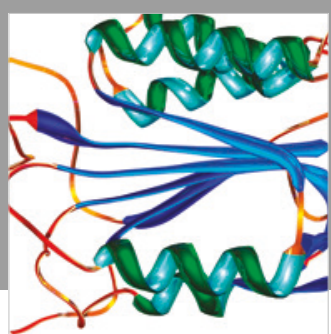

Disease Markers
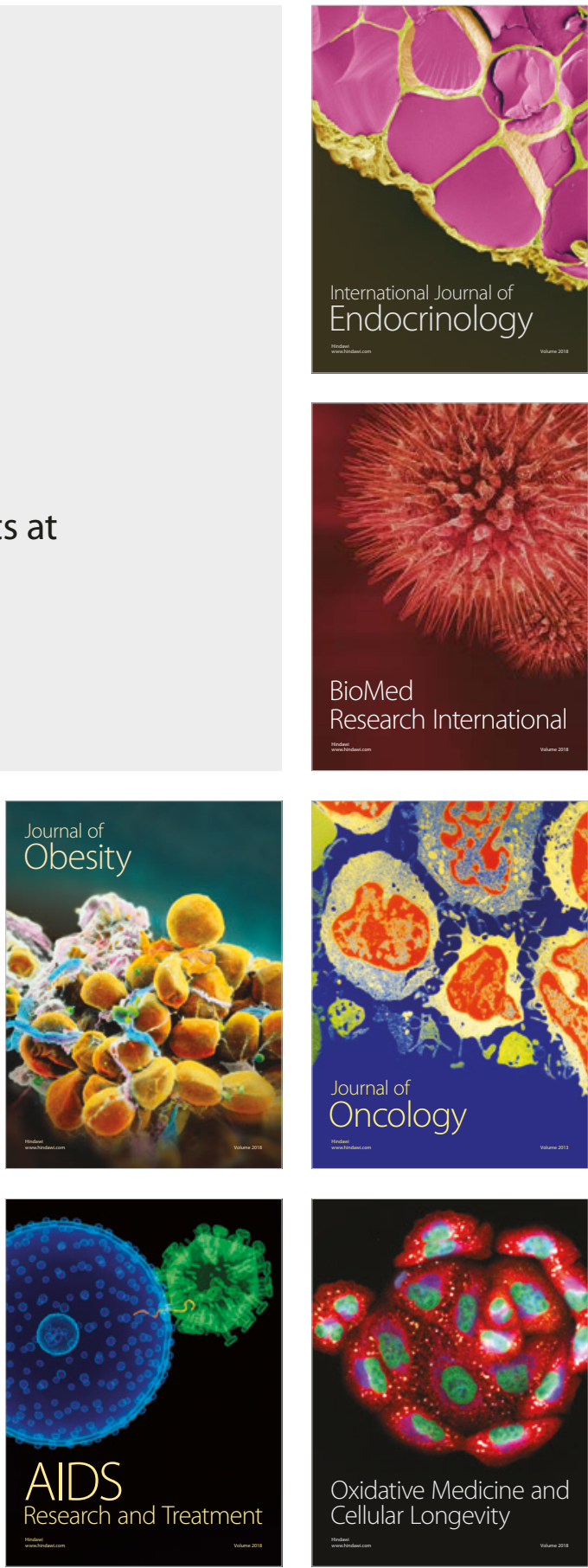